\title{
Effects of Topical Nipradilol on Early Diabetic Retinopathy in SDT Rats
} \author{
Motoshige Kudo ${ }^{4}$, Masanobu Kawakami ${ }^{1}$ and Yasunori Kanazawa ${ }^{5}$ \\ ${ }^{1}$ Saitama Medical Center, Jichi Medical University, Saitama, Japan \\ ${ }^{2}$ Medical Affairs Department, Kowa Pharmaceutical Company, Ltd., Japan \\ ${ }^{3}$ Center of Molecular Medicine, Jichi Medical University, Shimotsuke, Japan \\ ${ }^{4}$ Department of Pathology, Tokyo Medical University, Tokyo, Japan \\ ${ }^{5}$ Japanese Diabetes Foundation, Tokyo, Japan
}

Nozomi Kinoshita ${ }^{1}$, Akihiro Kakehashi ${ }^{*}, 1$, Yoh Dobashi ${ }^{1}$, Ryuichiro Ono ${ }^{1}$, Fumihiko Toyoda ${ }^{1}$, Chiho Kambara $^{1}$, Hiroko Yamagami ${ }^{1}$, Yusuke Kitazume ${ }^{2}$, Eiji Kobayashi ${ }^{3}$, Yasuhiro Osakabe ${ }^{4}$,

\begin{abstract}
We tested the effect of topical nipradilol, an antiglaucoma drug with $\alpha$ - and $\beta$-blocker and nitric oxide (NO) donor activities, on the early retinal changes using electron microscopy in Spontaneously Diabetic Torii (SDT) rats. In seven young male SDT rats (17 to 18 weeks old), nipradilol solution was instilled in the right eyes and nipradilol-free base solution in the left eyes three times daily for 3 months. All rats were sacrificed and both eyes were enucleated for electron microscopy at the end of the experiments. All rats had blood glucose levels exceeding $350 \mathrm{mg} / \mathrm{dl}$ within 2 weeks after the beginning of the experiment (mean final blood glucose level, $558 \pm 100.2 \mathrm{mg} / \mathrm{dl}$ ). In untreated eyes of young SDT rats, the overall pathological features were almost comparable to those in older SDT rats, although the pinocytotic vesicles and free ribosomes were not as remarkable in the latter. In contrast, in nipradilol-treated eyes of SDT rats, although the basement membrane was thickened, microvilli were seen, and a larger number of electron-dense mitochondria were in the wide cytoplasm. Lipofuscin-like electron-dense granules and lamellated myelin figures also were identified. Nipradilol reduced the early morphologic changes in the endothelial cells, which reflects the metabolically active state of diabetic retinopathy in SDT rats through its action as a NO donor.
\end{abstract}

Keywords: Nipradilol, $\alpha$ - and $\beta$-blocker, nitric oxide (NO) donor, SDT rat, diabetic retinopathy, electron microscopy.

\section{INTRODUCTION}

The Spontaneously Diabetic Torii (SDT) rat is a novel animal model of proliferative diabetic retinopathy (DR). Large retinal folds of thickened retina with vitreous traction and extensive dye leakage around the optic disc combined with vascular tortuosity by fluorescein microangiography are the most typical characteristics of advanced retinopathy in SDT rats (Fig. 1). The prevalence of advanced retinopathy in SDT rats is about $80 \%$ by 60 weeks of age, but advanced retinopathy is rarely seen before 50 weeks of age [1]. Microscopic changes of DR can be observed much earlier than those macroscopic changes. Microscopic changes of DR in old SDT rats include basement membrane (BM) thickening in the form of reduplication caused by invagination of the cytoplasmic process into the membrane. Furthermore, in the endothelial cells, clear mitochondria and dilated rough endoplasmic reticulum are abundant. Those findings are not observed in old normal non-diabetic SD rats. Thus, these morphologic changes are not age-related but caused by diabetes (Fig. 2) [2].

*Address correspondence to this author at the Department of Ophthalmology, Saitama Medical Center, Jichi Medical University, 1-847 Amanuma, Omiya, Saitama, 330-8503, Japan; Tel: +81-48-647-2111; Fax: +81-48-6485188; E-mail: kakeaki@omiya.jichi.ac.jp
In early-stage DR, electrophysiologic changes such as the absence of oscillatory waves in the electroretinogram are seen even with no funduscopic changes. Previous studies showed that leukocyte adhesion to the retinal endothelial cells initiated DR [3-6]. Using acridine orange leukocyte digital fluorography, we previously reported that topical nipradilol, an antiglaucoma agent with $\alpha$ - and $\beta$-blocker and nitric oxide (NO) donor activity (3,4-dihydro-8-(2-hydroxy3 -isopro-pylamino) propoxy-3-nitroxy-2H-1-benzo-pyran; molecular weight, 326.34) (Fig. 3) significantly reduced leukocyte adhesion to the endothelium of retinal vessels in early-phase DR in streptozotocin (STZ)-induced diabetic rats [7].

In the current study, we tested the effect of nipradilol on early retinal changes in SDT rats using electron microscopy.

\section{METHODS}

All procedures were performed according to the Association for Research in Vision and Ophthalmology (ARVO) Statement for the Use of Animals in Ophthalmic and Vision Research and approved by the Jichi Medical University Animal Care and Use Committee.

\section{Animals}

Seven young male SDT rats (17 to 18 weeks old, CLEA, Inc., Tokyo, Japan) were used. Nipradilol 1\% ophthalmic 

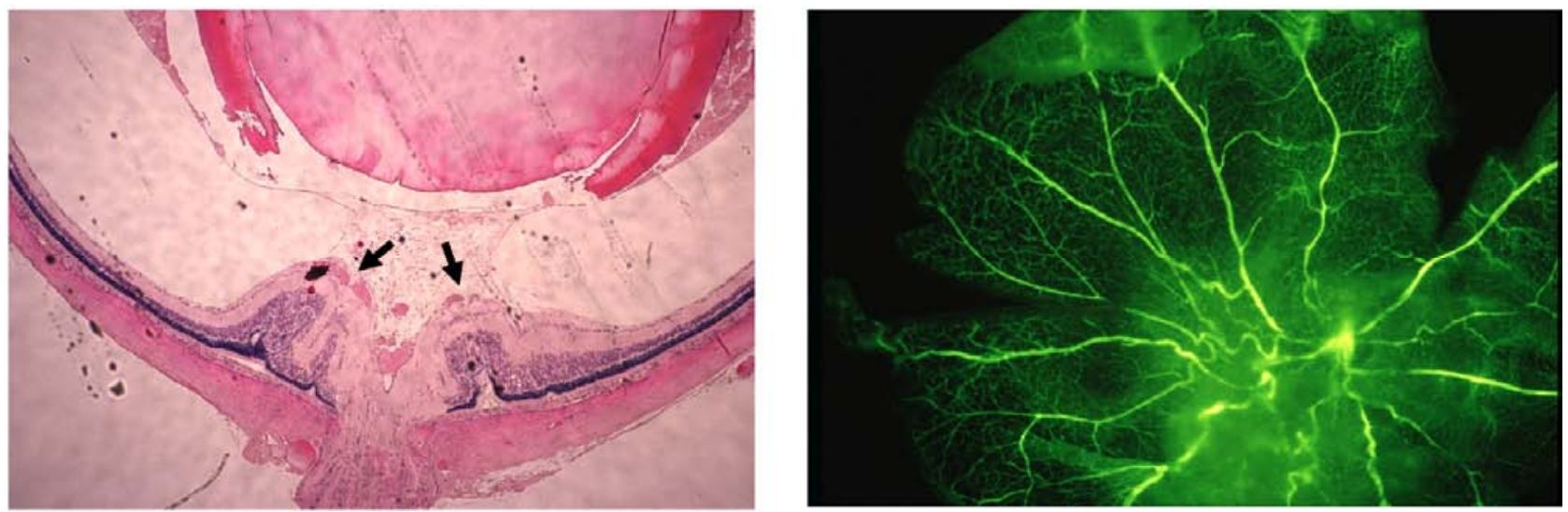

Fig. (1). Proliferative DR in SDT rats. Large retinal folds of thickened retina with vitreous traction (arrows) are the most typical characteristics of advanced retinopathy (left). Fluorescein microangiography shows extensive dye leakage around the optic disc with vascular tortuosity (right) (modified from Kakehashi [2]) in SDT rats over 50 weeks of age.

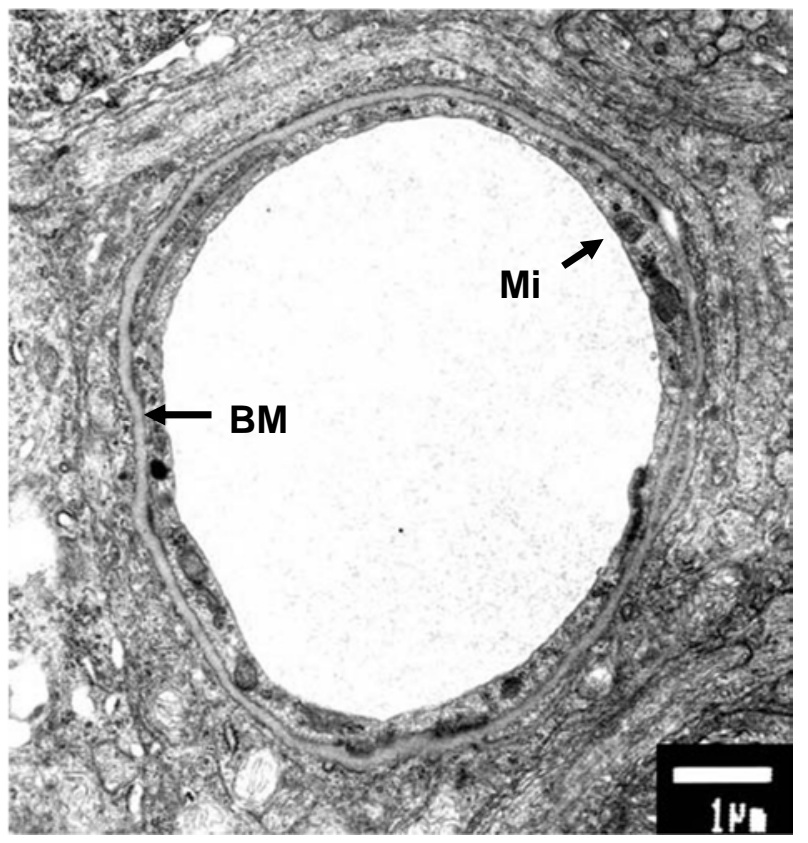

42-week-old normal male SD rat

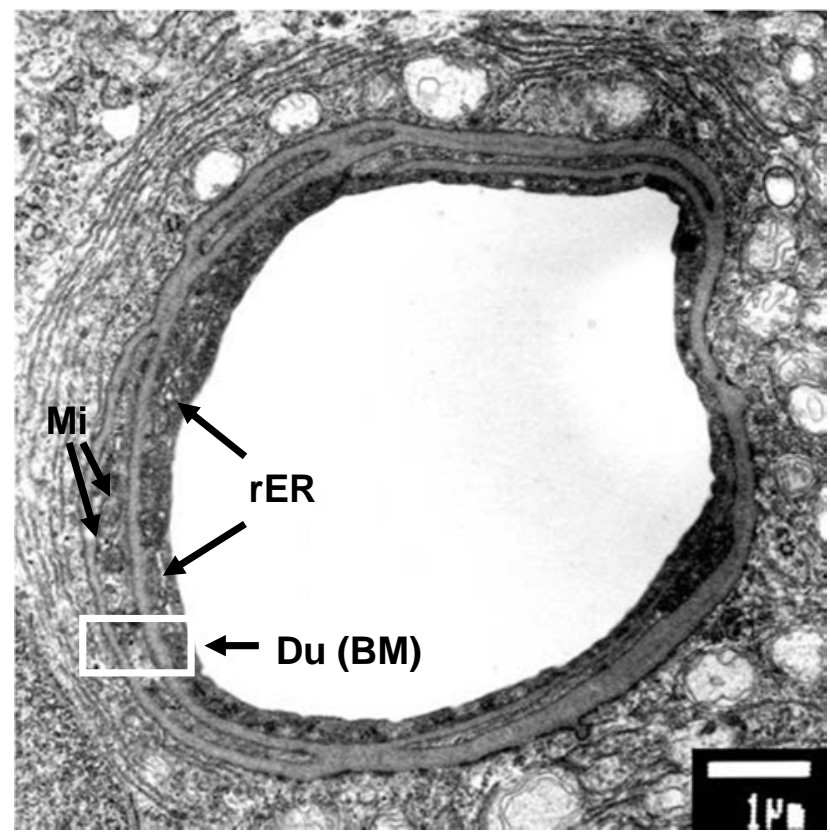

42-week-old male SDT rat

Fig. (2). Electron microscopic findings in the retinal capillary in older SD rats and SDT rats (modified from Kakehashi [2]). Basement membrane (BM) thickening by invagination of the endothelial cytoplasm is prominent in an older untreated SDT rat (right) compared with the thin clean membrane in a normal SD rat (left). Clear mitochondria (Mi) and dilated rough endoplasmic reticulum (rER) and reduplication $(\mathrm{Du})$ of the $\mathrm{BM}$ are seen in an older untreated SDT rat (right).<smiles>CC(C)NCCO</smiles>

Fig. (3). Nipradilol. Nipradilol (3,4-dihydro-8-(2-hydroxy-3isopropylamino)propoxy-3-nitroxy-2H-1-benzopyran; molecular weight, 326.34) has not only antiglaucomatous effects that depend mainly on selective $\alpha 1$-receptor and nonselective $\beta$-receptor blocking activity to reduce intraocular pressure but also NO donor activity through its nitroxyl group. solution (Kowa Pharmaceutical Company, Ltd., Japan) was instilled in the right eyes and nipradilol-free base solution was instilled in the control left eyes three times daily for 3 months. Blood glucose levels were measured at baseline, 2 weeks, and 1, 2, and 3 months after the start of treatment.

\section{Procedure to Observe Early Retinal Changes in SDT Rats}

All rats were sacrificed and both eyes were enucleated about 3 months after the start of the experiment (about 28 weeks of age). We performed electron microscopy to compare the changes in retinal vasculature between nipradilol-treated eyes and untreated control eyes in the SDT rats. The anesthetized SDT rats were perfused via the left 
ventricle with $300 \mathrm{ml}$ of $2.5 \%$ glutaraldehyde in $0.2 \mathrm{M}$ phosphate buffer $(\mathrm{pH}, 7.4)$. The retina was dissected from the eye cup and post-fixed in phosphate buffered $1 \%$ osmium tetroxide $(\mathrm{pH}, 7.4)$, dehydrated in ascending concentrations of ethanol, and passed through n-butyl glycidyl ether. The retinal blocks were embedded in epoxy resin. Thin sections were cut and stained in uranyl and lead salt solutions and observed by electron microscopy (JEM1200EX, JEOL Ltd., Tokyo, Japan).

\section{RESULTS}

All rats were confirmed to have blood glucose levels exceeding $350 \mathrm{mg} / \mathrm{dl}$ within 2 weeks after the beginning of the experiment. The mean final blood glucose level 3 months after the beginning of the experiment was $558 \pm 100.2 \mathrm{mg} / \mathrm{dl}$.

When we observed retinal specimens from nipradiloltreated and nipradilol-untreated eyes using electron microscopy, the retinal capillaries had well-preserved ultrastructural features and many features of the endothelial cells, including the plasma membrane and basal lamina, could be identified.

In untreated eyes of young SDT rats (Fig. 4), BM thickening was apparent despite the younger age, and the morphology was almost identical to that of the older SDT rats (Fig. 2). Although the endothelial cells were plump, the cytoplasmic space was smaller compared with the older nondiabetic SD rats. In this relatively narrow cytoplasmic space, pinocytotic vesicles, clear mitochondria, dilated rough endoplasmic reticulum, and free ribosomes were remarkable.
Moreover, pericytes were generally shrunken and decreased in number. These overall features were almost comparable to those in older SDT rats, although the pinocytotic vesicles and free ribosomes were not as remarkable in the latter.

In contrast, in nipradilol-treated eyes of SDT rats (Fig. 5), the cytoplasmic space in the endothelial cells was broader despite the flattened morphology compared with those in untreated eyes (Fig. 4). Thus, the cytoplasmic organelles were seen more clearly. Although the pinocytotic vesicles were not abundant, microvilli were observed. The mitochondria were electron-dense, and the numbers were greater compared with those in untreated eyes. Another difference was the electron-dense granules, some of which were reminiscent of lipofuscin. Lamellated myelin figures also were seen. In the nipradilol-treated eyes, there were more and larger pericytes. The alterations of the BM were similarly observed, and no significant difference was found compared with those in the untreated eyes.

\section{DISCUSSION}

In early-stage DR, adhesion of leukocytes to the retinal endothelial cells (retinal leukostasis) is considered a key pathogenic process [3-7]. Furthermore, recent studies have suggested that vascular endothelial growth factor (VEGF) levels are increased not only in late-stage [8,9] but also in early-stage DR [10]. As a result of VEGF-induced leukocyte adhesion to the diabetic retinal vasculature, retinal vascular endothelial cells are affected by leukocytes, resulting in breakdown of the blood-retinal barrier and capillary nonperfusion [11]. In the alloxan-induced diabetic rat,
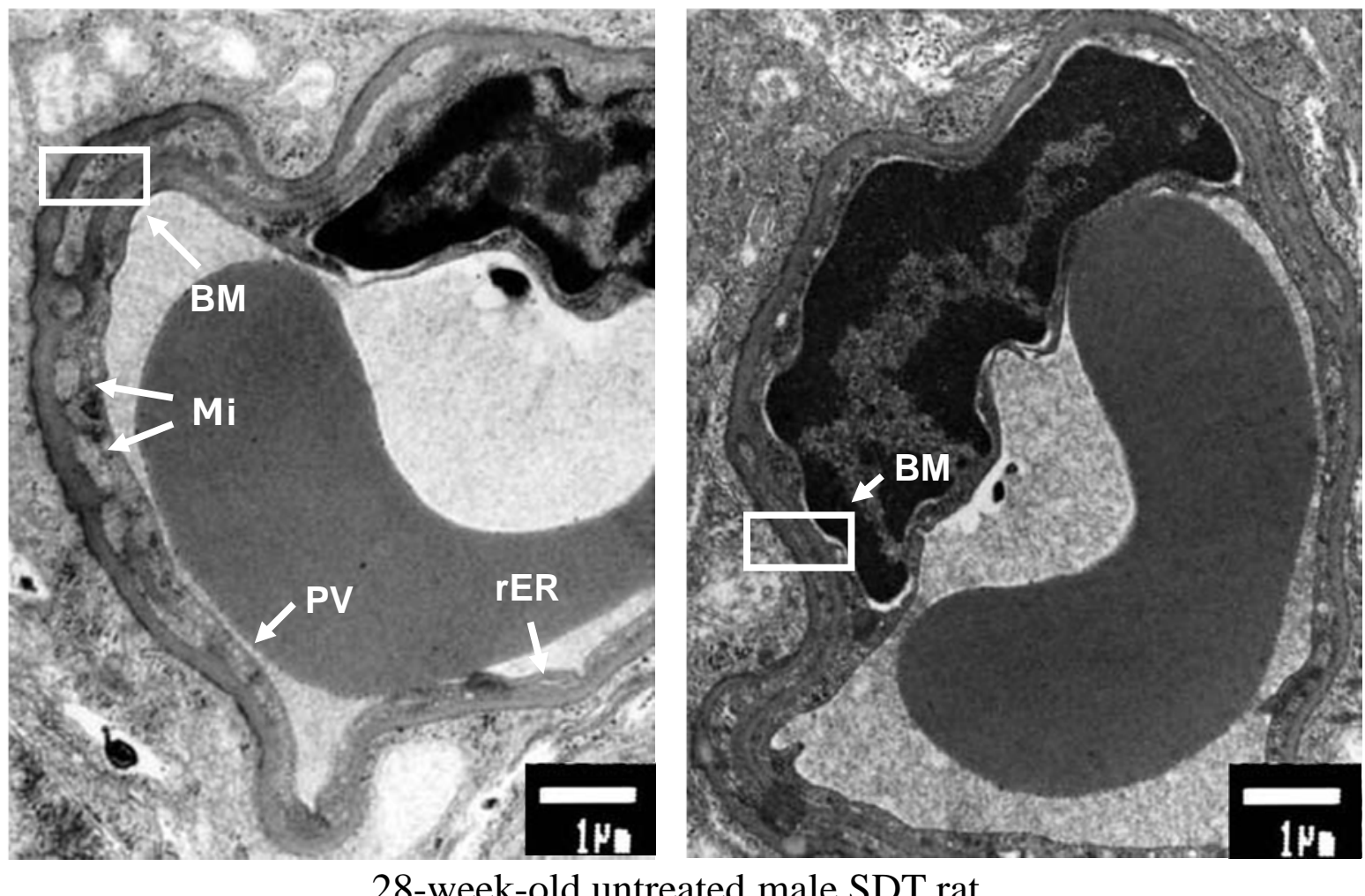

28-week-old untreated male SDT rat

Fig. (4). Electron microscopic findings of the retinal capillaries in a young untreated SDT rat. In the untreated group, BM thickening is apparent despite younger age. The endothelial cells of the retinal capillaries are plump but have narrow cytoplasm (right and left). Pinocytotic vesicles (PV), clear mitochondria (Mi), dilated rough endoplasmic reticulum (rER), and free ribosomes are remarkable (left). 

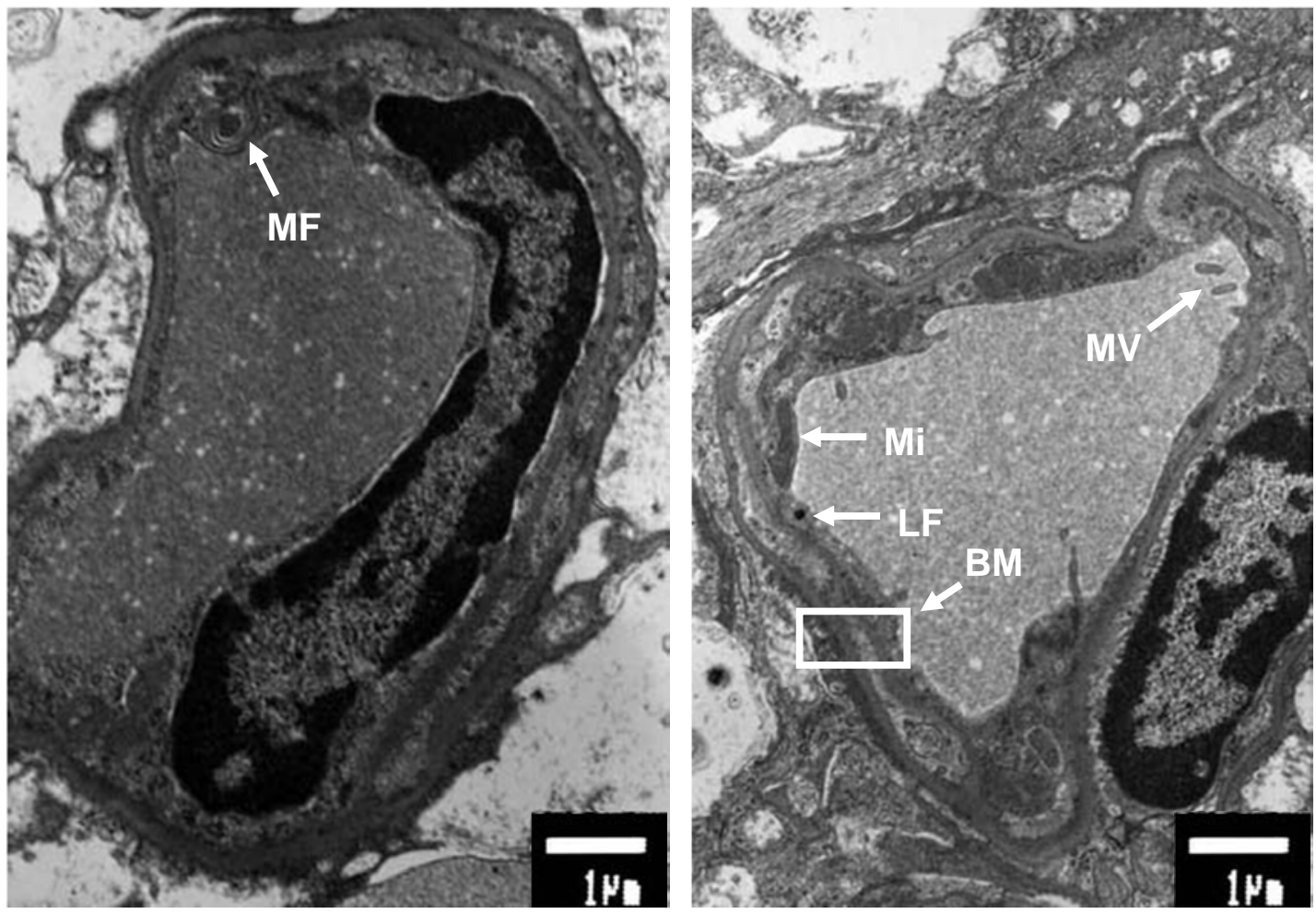

\section{8-week-old nipradilol-treated male SDT rat}

Fig. (5). Electron microscopic findings of the retinal capillaries in a young nipradilol-treated SDT rat. In the nipradilol-treated group, the endothelial cells in the retinal capillaries are flattened but have larger cytoplasm. Microvilli (MV) and electron-dense mitochondria (Mi) are seen (right). Electron-dense lipofuscin (LF)-like granules (right) and lamellated myelin figures (MF) (left) also are seen. The alterations of the basement membrane (BM) were similarly observed compared with those in the untreated eyes (left).

Schroder et al. reported that neutrophils and monocytes occluded retinal capillaries in histologic sections and that areas of endothelial cell damage were present around the leukocytes [12]. Therefore, preventing retinal leukostasis may be a possible therapeutic approach [6]

Both reduced retinal blood flow and vasoconstriction of retinal vessel progress in early-stage DR [13]. The important roles of $\mathrm{NO}$ in the retinal microcirculation, i.e., increased blood flow through nitroglycerine-like vasodilation and decreased microvascular occlusion through suppression of platelet coagulation and leukocyte adhesion to the endothelium, are well recognized [14]. Nipradilol works as a NO donor agent through its nitroxyl moiety in the molecule in addition to $\alpha$ - and $\beta$-blocking effects (Fig. 3). We reported that topical nipradilol significantly suppressed retinal leukostasis [7], suggesting that the NO derived from nipradilol inhibits leukocyte adhesion to the vascular endothelium.

It is well known that pericyte loss, BM thickening, and proliferation of endothelial cells occur in the retinal capillaries of STZ-induced diabetic rats and mice [15-17] and humans with diabetes [18]. However, the initial changes in early DR are still uncertain. The endothelial changes and subsequent changes in the BM seem to be the initial changes in early DR in SDT rats.

In the current study, pathological changes were observed in the BM, endothelial cells, and pericytes in SDT rats, which are uncommon findings in old non-diabetic SD rats, indicating that those findings are diabetes-related rather than age-related. Moreover, since BM changes also were seen in the nipradilol-treated group, morphologic BM changes seem to be established in early-phase diabetes and cannot be prevented. We also showed that the endothelial cells of the retinal capillaries were plump with smaller cytoplasm in untreated eyes. Furthermore, the ultrastructural findings, such as abundant pinocytotic vesicles, dilated rough endoplasmic reticulum, and free ribosomes reflect the metabolically active state of the endothelial cells. The decrease in the number of pericytes was consistent with a previous study [18]. Among these several findings, abundant pinocytotic vesicles and free ribosomes were more conspicuous. In turn, in nipradilol-treated eyes, the microvilli were preserved and electron-dense granules and presumably lipofuscin were prominent. The coexistence of lamellated myelin figures, often observed as lipofuscin derivatives, supports this. Considering the other findings, including fewer dilated rough endoplasmic reticulum, pinocytotic vesicles, and free ribosomes, these overall results suggested that the metabolic activity elicited and up-regulated by diabetes was suppressed after nipradilol treatment, and nipradilol may further cause "wear-and-tear changes" represented by the appearance of lipofuscin. Thus, endothelial cells may not functionally revert, but their proliferative capacity definitely is down-regulated by topical nipradilol, since these pathological changes in the 
endothelial cells decreased significantly in the treated eyes. These findings also may suggest that the NO activity of nipradilol protects the vascular endothelial cells by suppressing retinal leukostasis.

Mizuno et al. reported that nipradilol reaches the periocular tissues around the optic disc in rabbit eyes at an effective concentration 60 minutes after topical instillation [19]. Geroski and Edelhauser reported that nipradilol may reach the retina-choroid through the periocular transscleral route, since the molecular weight of nipradilol is as small as 326.34 [20, 21]. Therefore, topical nipradilol may contribute NO to the retinal circulation.

\section{CONCLUSION}

In conclusion, the pathological changes of the BM in DR are established in the early phase and treatment with topical nipradilol did not prevent them. However, nipradilol reduced the early morphologic changes in the endothelial cells, which reflects the metabolically active state of DR in SDT rats. Therefore, this drug may have prophylactically prevented development of proliferative changes in the endothelial cells in DR through its activity as a NO donor. In the future, our next experimental work will clarify whether nipradilol can prevent advanced DR or not.

\section{REFERENCES}

[1] Kakehashi A, Saito Y, Mori K, et al. Characteristics of diabetic retinopathy in SDT rats. Diabetes Metab Res Rev 2006; 22: 45561.

[2] Kakehashi A. Pathogenesis of diabetic retinopathy: Lessons from diabetic animal model. Endocrinol Diabetol 2003; 17:31-7 (Japanese).

[3] Abiko T, Abiko A, Clermont AC, et al. Characterization of retinal leukostasis and hemodynamics in insulin resistance and diabetes: role of oxidants and protein kinase-C activation. Diabetes 2003; 52: 829-37.

[4] Kinoshita N, Kakehashi A, Inoda S, et al. Effective and selective prevention of retinal leukostasis in streptozotocin-induced diabetic rats using gliclazide. Diabetologia 2002; 45: 735-9.

[5] Miyamoto K, Hiroshiba N, Tsujikawa A, Ogura Y. In vivo demonstration of increased leukocyte entrapment in retinal microcirculation of diabetic rats. Invest Ophthalmol Vis Sci 1998; 39: 2190-4.
[6] Miyamoto K, Khosrof S, Bursell SE, et al. Prevention of leukostasis and vascular leakage in streptozotocin-induced diabetic retinopathy via intercellular adhesion molecule-1 inhibition. Proc Natl Acad Sci U S A 1999; 96: 10836-41.

[7] Ono R, Kakehashi A, Ito Y, et al. Effect of topical nipradilol on retinal microvascular leukocyte adhesion in diabetic rats. Ophthalmic Res 2006; 38: 270-3.

[8] Adamis AP, Miller JW, Bernal MT, et al. Increased vascular endothelial growth factor levels in the vitreous of eyes with proliferative diabetic retinopathy. Am J Ophthalmol 199415; 118: 445-50.

[9] Aiello LP, Avery RL, Arrigg PG, et al. Vascular endothelial growth factor in ocular fluid of patients with diabetic retinopathy and other retinal disorders. N Engl J Med 1994; 331: 1480-7.

[10] Tanaka Y, Katoh S, Hori S, Miura M, Yamashita H. Vascular endothelial growth factor in diabetic retinopathy. Lancet 1997; 349(9064): 1520.

[11] Qaum T, Xu Q, Joussen AM, et al. VEGF-initiated blood-retinal barrier breakdown in early diabetes. Invest Ophthalmol Vis Sci 2001; 42: 2408-13.

[12] Schroder S, Palinski W, Schmid-Schonbein GW. Activated monocytes and granulocytes, capillary nonperfusion, and neovascularization in diabetic retinopathy. Am J Pathol 1991; 139: $81-100$.

[13] Wright WS, Harris NR. Ozagrel attenuates early streptozotocininduced constriction of arterioles in the mouse retina. Exp Eye Res 2008; 86: 528-36.

[14] Dorner GT, Garhofer G, Kiss B, et al. Nitric oxide regulates retinal vascular tone in humans. Am J Physiol Heart Circ Physiol 2003 285: H631-6.

[15] Kato N, Yashima S, Suzuki T, Nakayama Y, Jomori T. Long-term treatment with fidarestat suppresses the development of diabetic retinopathy in STZ-induced diabetic rats. J Diabetes Complications 2003; 17: 374-9.

[16] Song E, Dong Y, Han LN, et al. Diabetic retinopathy: VEGF, bFGF and retinal vascular pathology. Chin Med J (Engl) 2004; 117: $247-51$

[17] Li Q, Verma A, Han PY, et al. Diabetic eNOS knockout mice develop accelerated retinopathy. Invest Ophthalmol Vis Sci 2010; 51: 5240-6.

[18] Bek T. Epidemiology of Diabetic Retinopathy. 2nd ed. van Bijsterveld OP, Ed. London: Martin Dunitz Ltd 2000.

[19] Mizuno K, Koide T, Yoshimura M, Araie M. Neuroprotective effect and intraocular penetration of nipradilol, a beta-blocker with nitric oxide donative action. Invest Ophthalmol Vis Sci 2001; 42: 688-94.

[20] Geroski DH, Edelhauser HF. Drug delivery for posterior segment eye disease. Invest Ophthalmol Vis Sci 2000; 41: 961-4.

[21] Geroski DH, Edelhauser HF. Transscleral drug delivery for posterior segment disease. Adv Drug Deliv Rev 2001; 52: 37-48. 